\title{
Geistes-Gegenwart. Rede und Vorlesung bei Adam Müller
}

Das 18. Jahrhundert entdeckte die Gegenwart. Oder genauer: Es gab dem Konzept der ,Gegenwart‘ eine neue Bedeutung. Denn selbstverständlich sprach man schon zuvor von der ,Gegenwart', doch damit wurde allein auf räumliche Relationen Bezug genommen. Gegenwärtig war das, was sich in unmittelbarer Nähe befand. Die zeitliche Dimension von Gegenwart aber - die für die moderne Semantik des „vielfach merkwürdige[n] wortes“1 ${ }^{\text {"1 }}$ zentral geworden ist - spielte vor der zweiten Hälfte des 18. Jahrhunderts noch keine Rolle. ${ }^{2}$ Dieser Befund lässt sich anschaulich belegen durch einen Blick in die zeitgenössischen Lexika und Wörterbücher, sei es nun Zedlers Universal-Lexicon ${ }^{3}$ oder aber auch noch Adelungs Wörterbuch, wo als Äquivalent für „Gegenwart“ das Wort „Anwesenheit“ genannt wird. ${ }^{4}$ Adelungs Beleg stammt aus den 1790er Jahren, was sogar darauf hindeuten würde, dass die Verzeitlichung der Gegenwart erst zu Beginn des 19. Jahrhunderts eingesetzt hätte. Denn erst dann wird tatsächlich auch in den Wörterbüchern die temporale Semantik von ,Gegenwart‘ verbucht; so etwa bei Campe, der 1808 neben der räumlichen auch die zeitliche Bedeutung nennt und „Gegenwart“ als „die gegenwärtige Zeit“ bestimmt. ${ }^{5}$

Doch Lexika und Wörterbücher hinken der tatsächlichen semantischen Entwicklung der Sprache notorisch hinterher. Sie haben sozusagen ein problematisches Verhältnis zum Sprachgebrauch ihrer jeweiligen Gegenwart als „gegenwärtige[r] Zeit“. Und entsprechend ist es auch im Falle des Konzepts der verzeitlichten Gegenwart so, dass schon rund 30 bis 40 Jahre vor Campe erste Belege für die neue Bedeutung des Wortes gefunden werden können. ${ }^{6}$

\footnotetext{
1 Grimm 1897: 2281.
}

2 Vgl. ebd.: 2291; ebenso Lehmann 2016: insb. 59-61; allgemeiner Oesterle 1985.

3 Vgl. o.A. 1735: 594.

4 Adelung 1796: 488.

5 Campe 1808: 265: „Die Gegenwart, [...] 1) Der Zustand, da man in eigner Person an einem Orte ist, die Anwesenheit. [...] 2) Die gegenwärtige Zeit.“ Belegt mit folgendem Schiller-Zitat: „Und blicket froher in die Gegenwart“ (ebd.). Dieses Zitat stammt aus dem 1798 verfassten und 1800 publizierten Prolog der Wallenstein-Trilogie und lautet im Zusammenhang: „Noch einmal laßt des Dichters Phantasie / Die düstre Zeit [des Dreißigjährigen Krieges] an euch vorüberführen, / Und blicket froher in die Gegenwart / Und in der Zukunft hoffnungsreiche Ferne." (Schiller 1993a: 272, V. 75-78)

6 Ganz vereinzelt lassen sich auch noch ältere Belege finden. Doch das sind extreme Ausnahmen, die die Regel bestätigen, wonach das Substantiv ,Gegenwart‘ erst seit den 1770er Jahren 
So wird zum Beispiel in der 1776 erschienenen deutschen Übersetzung von Louis-Sébastien Merciers Du théâtre, ou nouvel essai sur l'art dramatique (1773) gefordert, dass Dramen einen „Karakter von Nützlichkeit für die Gegenwart [caractère d'utilité présente]" haben sollten, ${ }^{7}$ was nur möglich sei, wenn die Autoren sich von den antiken Vorbildern lösten und sich auf ein vertieftes Studium „der jetzigen Menschen [des hommes actuels]“ einließen. ${ }^{8}$ Mit impliziert in dieser Forderung ist die für die zweite Hälfte des 18. Jahrhunderts grundlegende Erkenntnis, dass die historisch-soziale Entwicklung nicht mehr anhand tradierter Muster und Beispiele angemessen erfasst werden kann. ${ }^{9}$ Vielmehr entwickeln sich Politik, Wirtschaft, Kultur und Gesellschaft in einem zukunftsoffenen Prozess, in dem jedes Zeitalter, jede Generation, sozusagen aus sich selbst heraus die Maßstäbe entwickeln muss, die eine Orientierung in der je eigenen, von allen früheren Epochen notwendig unterschiedenen Gegenwart ermöglichen. Für das Theater, von dem Mercier spricht, heißt das, dass es in seiner jeweiligen Gegenwart auf die Fragen, Nöte und Hoffnungen eben jener Gegenwart Bezug nehmen muss.

Was aber vom Theater gilt, gilt mutatis mutandis auch von der Wissenschaft. Oder anders gewendet: Wenn Schiller 1784 in Mannheim fragte: „Was kann eine gute stehende Schaubühne eigentlich wirken?"“, ${ }^{10}$ so stellte sich auch für die Wissenschaft mit neuer Virulenz die Frage, was sie in ihrer jeweiligen Gegenwart - nun eben verstanden im emphatischen zeitlichen Sinne - wirken könne und wie sie es könne. Ja man kann im Grunde das ganze Ringen um eine angemessene Wissenschaftssprache und um geeignete Darstellungsformen für die Wissensvermittlung als ein Ringen um Gegenwärtigkeit im Sinne eines Gegenwartsbezugs deuten - und dies auf mehreren Ebenen, auf denen sich, wie $\mathrm{zu}$ zeigen sein wird, die neue zeitliche Bedeutungsdimension von Gegenwart

in zeitlichem Sinne benutzt wird. So verzeichnet Trübners Deutsches Wörterbuch einen Beleg von 1745, vgl. o.A. 1939: 59, und das Frühneuhochdeutsche Wörterbuch sogar einen von 1466, vgl. o.A. 2005: 517.

7 Mercier 1776: 199 resp. Mercier 1773: 149. Den Hinweis auf diesen Beleg verdanke ich Lehmann 2016: $57 \mathrm{f}$.

8 Mercier 1776: 198 resp. Mercier 1773: 149. Die entsprechende Passage lautet dort im Zusammenhang: „On a étudié les anciens, \& l'on a bien fait; mais ce n'est pas chez eux qu'on trouvera une connoissance détaillée des hommes actuels: De nouvelles générations ont apporté de grands changemens dans cet être moral [d.h. im Menschen], dans ce prothée [Proteus], qui en échappant revêt toutes les formes [...]. / Il reste à imprimer au Drame un caractère d'utilité présente, la connoissance de l'homme \& des choses avantageuses à la société.“

9 Vgl. dazu prominent Koselleck 1989; ebenso Luhmann 1980.

10 Schiller 1993c. 
auf unterschiedliche Art und Weise mit der älteren räumlichen Bedeutung amalgamieren konnte.

Relativ offensichtlich ist das Bemühen um Gegenwärtigkeit im Zusammenhang mit der im 18. Jahrhundert erfolgten Ablösung des Lateinischen als Wissenschaftssprache durch das Deutsche. ${ }^{11}$ Wer von seinen Zeitgenossen gehört und gelesen werden wollte, durfte sich nicht mehr der Gelehrtensprache der Vergangenheit bedienen. Aber auch die in der Einleitung zum vorliegenden Band so konzis beschriebene epistemologische Zäsur, die sich im Wandel des Konzepts von Gelehrsamkeit beobachten lässt, steht in unmittelbarem Zusammenhang mit der damals mit Nachdruck gestellten Frage nach dem Gegenwartsbezug der Wissenschaft. Eine Wissenschaft, die für ihre Gegenwart einen - um mit Mercier zu sprechen - „Karakter von Nützlichkeit“ haben wollte, konnte sich nicht mehr auf überlieferte Autoritäten verlassen, sondern musste dynamisch auf den stetigen und zukunftsoffenen Wandel im historischen Prozess reagieren. Das hieß, dass Vorlesungen sich nicht mehr in der Überlieferung von Lehrbuchwissen erschöpfen durften. Vielmehr sollte das tradierte Wissen - wie beispielsweise Schelling paradigmatisch formulierte - „durch eigne Erfindungen“ bereichert und „mit Geist“ in „lebendige[m] Vortrag“ vermittelt werden. ${ }^{12}$ Und zudem musste die Wissenschaft, wollte sie tatsächlich auf ihre Gegenwart wirken, immer wieder neu darum ringen, Darstellungsformen zu finden, die es ihr erlaubten, nicht nur den exklusiven Kreis der Gelehrten, sondern auch ein weiteres Publikum zu erreichen.

Was es brauchte, war eine neue Rhetorik. Das mag zunächst befremdlich klingen, da üblicherweise der ,Tod der Rhetorik‘ auf das Ende des 18. Jahrhunderts datiert wird und sich das movere der klassischen Rhetorik zumindest nach Kant'schen Kriterien kaum mit dem docere des wissenschaftlichen Lehrvortrags vereinbaren lässt. ${ }^{13}$ Doch eine Rhetorikgeschichtsschreibung, die sich, wie es so oft geschieht, zu stark an Kant als Dreh- und Angelpunkt orientiert, verfehlt wichtige Entwicklungen der Jahrzehnte um $1800 .{ }^{14}$ Denn auch wenn

11 Vgl. zu diesem Übergang Hambsch 2009: 1195.

12 Schelling 1803: 46. Geschehe das nicht, fügt er an, sei nicht zu begreifen, „wofür nur überhaupt der lebendige Vortrag auf Academieen nothwendig wäre; man könnte alsdann den Lehrling unmittelbar nur an die ausdrücklich für ihn geschriebenen, gemeinfaßlichen Handbücher oder an die dicken Compilationen in allen Fächern verweisen“ (ebd.). Vgl. z. B. auch Schleiermacher: „[I]hm [dem Meister der Wissenschaft] wird keine Wiederholung möglich sein, ohne daß eine neue Kombination ihn belebt, eine neue Entdeckung ihn an sich zieht; er wird lehrend immer lernen, und immer lebendig und wahrhaft hervorbringend dastehn vor seinen Zuhörern.“ (Schleiermacher 1996: 373)

13 Vgl. zu Kants Kritik an der ars oratoria v. a. Kant 1974: 258f., 266f. (§ 51 und 53).

14 Vgl. dazu Schnyder 2005. 
die Rhetorik damals als umfassendes Lehrsystem zweifellos an Bedeutung verlor, erlebte sie doch in manchen Bereichen in den deutschsprachigen Ländern überhaupt erst nach 1800 einen Aufschwung; so etwa in der Rechtsprechung (mit den erst damals eingeführten öffentlich-mündlichen Verfahren), in der Politik, in der Homiletik - oder eben auch in der Wissenschaft.

Einer nun, der damals mit besonderer Verve für eine Erneuerung der deutschen Rhetorik eintrat, war Adam Müller, dessen Zwölf Reden über die Beredsamkeit und deren Verfall in Deutschland - gehalten 1812 in Wien, publiziert 1816 in Leipzig - im Fokus der folgenden Ausführungen stehen werden. Für Müller war die Redekunst, wie er mit einem auffallenden Superlativ formulierte, die „gegenwärtigste unter allen Künsten“, ${ }^{15}$ und es wird darum gehen, diesen Superlativ in vier Unterabschnitten näher zu erörtern und insbesondere im Hinblick auf das - gerade in der Romantik wichtige, aber in der Literaturwissenschaft bis heute stiefmütterlich behandelte - Genre der öffentlichen wissenschaftlichen Vorlesung hin zu profilieren: ${ }^{16}$ Zunächst soll etwas über den konkreten historischen Ort und das performative Setting der Zwölf Reden gesagt werden (1. Die Gegenwart der Zwölf Reden). Danach wird das intrikate Verhältnis von Mündlichkeit und Schriftlichkeit in Müllers „gegenwärtigste[r]“ Kunst thematisiert; ein Verhältnis, in dem auch die ältere räumliche Bedeutung von ,Gegenwart' neu verhandelt wird (2. Das Medium der Gegenwart). Drittens wird die von Müller postulierte Fähigkeit des guten Redners behandelt, in jedem Augenblick seines Vortrags spontan auf die Reaktionen seines Publikums eingehen zu können (3. Geistesgegenwart). Und viertens schließlich soll die nicht nur von Müller geteilte Ansicht erörtert werden, wonach im lebendigen Vortrag neben den zwei Instanzen des Sprechers und des Publikums immer auch noch ein Dritter präsent sei, ein das Vortragsgeschehen durchwaltender und umfassender Geist, der in Müllers Konzept auf symptomatische Art und Weise zwischen dem Geist der Nation und dem Geist Gottes oszilliert (4. Die Gegenwart des Geistes).

15 Müller 1967a: 374, Hvh. P.S. Nachweise hieraus im Folgenden mit Angabe der Seitenzahl direkt im Text.

16 Symptomatisch für die Vernachlässigung dieses Genres in der Literaturwissenschaft ist zum Beispiel, dass es im in den Jahren 1997-2003 erschienenen Reallexikon der deutschen Literaturwissenschaft kein Lemma „Vorlesung“ gibt. Ähnlich sieht es in den anderen Philologien aus. Vgl. allerdings (mit expliziter Benennung des Forschungsdesiderats) Dick \& Esterhammer 2009. Wenn das Phänomen der öffentlichen Vorlesung im deutschsprachigen Kontext vereinzelt thematisiert wurde, geschah dies meist aus der Perspektive der Rhetorikforschung, vgl. Riedl 1997: 214-294; ebenso Hambsch 2009. 


\section{Die Gegenwart der Zwölf Reden}

Als der 1779 geborene Adam Müller 1811, also 32-jährig, nach Wien kam, war er kein unbeschriebenes Blatt mehr: ${ }^{17}$ Nach seinem Studium der Rechtswissenschaften und der Geschichte in Göttingen hatte er sich bald - auch dank der Unterstützung seines Freundes Friedrich Gentz - einen Namen gemacht mit Vorlesungszyklen in Dresden, wo er auch gemeinsam mit Heinrich von Kleist die Zeitschrift Phöbus herausgab. Daneben hatte er für einen handfesten gesellschaftlichen Skandal gesorgt, weil Sophie von Haza - die Frau von Peter Boguslaus von Haza-Radlitz, bei dem Müller in jenen Jahren als Hauslehrer angestellt war - sich scheiden ließ, um ihn zu heiraten. 1809 hatte er Sachsen dann aus politischen Gründen verlassen müssen und in Berlin eine rege politischpublizistische Tätigkeit aufgenommen, nicht zuletzt wiederum zusammen mit Kleist als Mitherausgeber der Berliner Abendblätter. Nachdem er sich allerdings in eben jenen Abendblättern kritisch über die Stein-Hardenberg'schen Reformen geäußert hatte, war er nach Wien geschickt worden - und Kleists Zeitung wurde einer so strengen Zensur unterstellt, dass ihr Erscheinen schließlich eingestellt werden musste. ${ }^{18}$

Wien nun war der Ort, wo Müller bereits einige Jahre zuvor, 1805, vom Protestantismus zum Katholizismus konvertiert war; ein Umstand, der seinen Ambitionen in Österreich zweifellos förderlich war. Trotzdem hatte er als Preuße in Wien keinen leichten Stand. Von Anfang an wurde er von der Polizei bespitzelt, ${ }^{19}$ und als er im Sommer 1811 bei den Behörden ein Gesuch einreichte, um „öffentliche Vorlesungen über das Ganze der Staatswissenschaften und die Lehre vom Gelde insbesondere“ anzubieten, erhielt er einen abschlägigen Bescheid. ${ }^{20}$ Das Thema war zu brisant für Österreich, das seit der Niederlage gegen Napoleon faktisch unter französischer Kontrolle stand und gerade einen Staatsbankrott hinter sich hatte. Im Frühling 1812 unternahm Müller dann - nicht zuletzt angeregt durch Friedrich Schlegels Vorlesungen über die Geschichte der alten und neuen Litteratur, die gerade damals in Wien stattfanden ${ }^{21}$ - einen

17 Vgl. für einen knappen biographischen Überblick die Ausführungen im Kommentar zu Müller 1967c: 301-311; ausführlicher Koehler 1980: 32-211.

18 Vgl. zu diesem wesentlich durch Müller mitverschuldeten Ende der Abendblätter Köhler 1980: $125 \mathrm{f} ., 149 \mathrm{f}$.

19 Diese Bespitzelung erfolgte auf Geheiß des Kaisers. Müllers Hausmeister erstattete regelmäßig Bericht an die Polizei, vgl. Köhler 1980: 182-184.

20 Vgl. dazu die Ausführungen im Kommentar zu Müller 1967c: 456.

21 Schlegel hielt diese Vorlesungen in Wien in der Zeit vom 27. Februar bis zum 30. April 1812; veröffentlicht wurden sie 1814, datiert auf 1815: Schlegel 1815. Vgl. dazu Polaschegg 2017: 224. 
neuen Versuch. Er wandte sich an die Polizeihofstelle und bat um die Erlaubnis, „außerordentliche Vorlesungen über die Rhetorik“ halten zu dürfen, also über einen, wie Müller formulierte, „völlig unschuldigen Gegenstand“.22 Nicht zu Unrecht zweifelten die Behörden allerdings an der Unschuld dieses Themas, denn schließlich war die Verbindung von Rhetorik und Politik schon seit der Antike topisch. Sie hatte im Kontext der Französischen Revolution neue Aktualität gewonnen und betraf keineswegs nur die politische Rhetorik im engeren Sinne. Vielmehr war, zumal nach dem Zusammenbruch des Heiligen Römischen Reichs Deutscher Nation im Jahre 1806, jedes Reden über deutsche Sprache und in deutscher Sprache zum Politikum geworden. ${ }^{23}$

Nach einigem Hin und Her und der Intervention von Gentz wurde der Bitte Müllers dennoch stattgegeben - freilich mit der geheimen Auflage, dass ein Spitzel die Vorlesungen kritisch begleiten sollte. ${ }^{24}$ So erschien im Oesterreichischen Beobachter vom 14. Mai 1812 die Ankündigung, dass der „Hofrat Adam Müller“ mit „allergnädigster Bewilligung Sr. k. k. Majestät“ am nächsten Tag „im k. k. Redoutengebäude“ eine Vorlesungsreihe eröffnen werde, für die in der „Schaumburgische[n] Buchhandlung in der Wollzeile [... .] Abonnements für 12 fl. W.W. [12 Gulden Wiener Währung]“ erworben werden könnten. ${ }^{25}$ Da Müller sich sorgte, dass der Wiener Adel, den er mit seinen Vorträgen vor allem im Blick hatte, die Stadt schon für die Sommerpause verlassen könnte, las er dann immer gleich dreimal pro Woche über die Mittagszeit von 12.15 bis 13 Uhr, „wobei“, wie es in der Ankündigung hieß, „um den Differenzen des Uhrenganges $\mathrm{zu}$ begegnen, die Uhr des Michaelsturms zur Richtschnur genommen" wurde. ${ }^{26}$

22 Müller an Freiherrn von Hager, den Vizepräsidenten der Polizeihofstelle, 1. April 1812, zit. nach Müller 1967c: 457.

23 Diese politische Bedeutung der deutschen Sprache ist in vielen zeitgenössischen Schriften offensichtlich, nicht zuletzt in Adam Müllers im Winter 1806 in Dresden gehaltenen Vorlesungen über die deutsche Wissenschaft und Literatur: „Waffen können in unglücklichen Augenblicken versagen; die Waffen des Geistes, sonderlich unsre teure unzerstörbare Sprache in ihrer biegsamen Kraft, verbleiben uns, und mit ihnen in aller äußern Not die lebendigste Hoffnung.“ (Müller 1967b: 21)

24 Vgl. dazu den Kommentar in Müller 1967c: 461f. Der abkommandierte Beamte erstattete dann tatsächlich mehrere geheime Berichte, die auf dem Dienstweg bis zu Metternich gelangten, der sie sogar an den Kaiser weiterleitete - freilich à contre cœur und nicht ohne darauf hinzuweisen, wie unqualifiziert ihm diese Berichte erschienen: „Warum unterlegt man endlich Allerhöchstdenselben [dem Kaiser Franz] solch elende Bruchstücke wie der vorliegende Polizei-Rapport.“ Zit. nach ebd.: 462.

25 O.A. 1812. Auch abgedruckt im Kommentar zu Müller 1967c: 458.

26 Ebd. 
Weshalb lohnt es sich, auf all diese Details zur Organisation von Müllers Vorlesungen einzugehen? - Nur über sie wird jenes performative Setting erkennbar, das nicht nur für Müllers und Friedrich Schlegels Vorlesungen, sondern überhaupt für eine bestimmte zeittypische Form des Redens über Wissenschaft signifikant ist. Es ist dies ein Reden, das seinen Ort nicht (oder jedenfalls nicht zwingend) in der Institution der Universität hat, sondern durchaus programmatisch den Brückenschlag zu einem allgemeineren Publikum - im Falle Müllers und Schlegels zu gesellschaftlich einflussreichen, meist adligen und zahlungskräftigen Kreisen versucht. ${ }^{27}$ Programmatisch ist dieser Brückenschlag, weil damit eben die Isolation der gelehrten Kommunikation durchbrochen und der Versuch unternommen wird, auf die eigene Gegenwart zu wirken.

Von dieser Wirkung auf die zeitliche Gegenwart, die durch die räumliche Gegenwart eines gemischten, auch weiblichen Publikums gleichsam inzitiert wird, spricht Müller denn auch schon in seiner ersten Vorlesung und macht zugleich deutlich, inwiefern seine Hörerinnen und Hörer für ihn ein umfassenderes Publikum repräsentieren, also im wörtlichen Sinne ,vergegenwärtigen': ${ }^{28}$

[S]olche öffentlichen Vorlesungen über Gegenstände der Wissenschaft oder der Kunst vor einer Versammlung von Personen, die weniger die Absicht, zu erlernen oder Kenntnisse zu erkaufen, als ein allgemeines, wahrhaft menschliches und gesellschaftliches Interesse an den Fortschritten der Bildung vereinigt, [sind] förderlich für die Belebung unsrer Sprache

27 Beispielhaft kommt dies in der Widmung an Metternich zum Ausdruck, die Schlegel seinen Vorlesungen im Druck vorangestellt hat: „[M]ein vorzüglichster Wunsch war es, der großen Kluft, welche immer noch die litterarische Welt und das intellektuelle Leben des Menschen von der praktischen Wirklichkeit trennt, entgegen zu wirken, und zu zeigen, wie bedeutend eine nationale Geistesbildung oft auch in den Lauf der großen Weltbegebenheiten und in die Schicksale der Staaten eingreift.“ (Schlegel 1815: o.P.) Vgl. zur entsprechenden Parallele zwischen Schlegel und Müller Herrmann 2015. Herrmann geht es freilich v. a. um die Umsetzung des genannten Programms in den Zeitschriftenprojekten der beiden Autoren und nur um am Rande um die Gattungen der Rede und der Vorlesung.

28 Dieser Gedanke der Vergegenwärtigung eines weiteren (Lese-)Publikums in der konkreten Hörerschaft findet sich z. B. auch in Fichtes im Winter 1807/1808 gehaltenen Reden an die deutsche Nation: „Sie [die Hörer] [. . . ] sind zwar meinem leiblichen Auge die ersten und unmittelbaren Stellvertreter, welche die geliebten Nationalzüge mir vergegenwärtigen, und der sichtbare Brennpunkt, in welchem die Flamme meiner Rede sich entzündet; aber mein Geist versammelt den gebildeten Teil der ganzen deutschen Nation, aus allen den Ländern, über welche er verbreitet ist, um sich her, bedenkt und beachtet unser aller gemeinsame Lage und Verhältnisse, und wünschet, daß ein Teil der lebendigen Kraft, mit welcher diese Reden vielleicht sie ergreifen, auch in dem stummen Abdrucke, welcher allein unter die Augen der Abwesenden kommen wird, verbleibe, und aus ihm atme, und an allen Orten deutsche Gemüter zu Entschluß und Tat entzünde.“ (Fichte 1997: 548) 
und überhaupt eine neue, sehr ehrenwerte Gattung in Deutschland [Hvh. P.S.]. Auch der [. . .] wissenschaftliche Teil unserer Literatur will also endlich gesellig werden: es soll nicht mehr ins Blaue und Unbestimmte hin, es soll nicht mehr den Wänden und Wüsten gepredigt werden, man will ein Lebendiges, ein Ganzes, eine würdige Stellvertretung des Publikums, zu dem man spricht, gegen sich über haben; [...] die deutsche Wissenschaft zeigt sich auf dem Wege nach einer großen Wahrheit, die bei uns mehr als irgendwo sonst vergessen worden ist, nämlich

daß es nur ein einziges Kennzeichen des Verständigen gebe, nämlich die Verständlichkeit, und daß man nur in demselben Grade selbst verstehe, als man verstanden wird. (300f.)

Das Medium der „deutsche[n] Wissenschaft“ auf dem Weg zu dieser „großen Wahrheit“ sind aber eben öffentliche Vorlesungen, eine „Gattung“, deren Neuheit in Deutschland Müller in der Folge noch weiter spezifiziert und dabei übrigens in ganz typischer Weise die deutsche ,Verspätung' gegenüber dem Westen mit einer Überbietungsgeste kompensiert: „Die größten wissenschaftlichen Autoritäten Deutschlands in und außer den hohen Schulen haben in den letzten zwanzig Jahren die Form solcher Vorlesungen gewählt und haben sie in dieser kurzen Zeit weiter ausgebildet, als es in Frankreich und England, wo sie längst in Gebrauch waren, gelungen ist“" (301).

Die Tradition des Redens über Wissenschaft, in die sich Müller stellt, hat also nach seiner eigenen Aussage erst in den 1790er Jahren begonnen, wobei als erstes Beispiel wohl an Fichtes Vorlesungen über die „Bestimmung des Gelehrten“ zu denken ist, die 1794 in Jena für Furore gesorgt hatten; ${ }^{29}$ vielleicht könnte man auch schon die Antrittsvorlesung Schillers ${ }^{30}$ von 1789 dazurechnen, den Müller in seinen Zwölf Reden als den „größten Redner“ Deutschlands feiert (302). Neben Fichte, der dann noch verschiedentlich inner- und außerhalb der Universität mit öffentlichen Vorlesungen hervorgetreten ist - die Reden an die deutsche Nation sind da ja nur die berühmtesten - sind auch Schleiermacher und Schelling sowie Friedrich und August Wilhelm Schlegel zu nennen; und nicht zuletzt natürlich Adam Müller selbst, der schon 1806 in Dresden einen überaus erfolgreichen Zyklus von Vorlesungen über die deutsche Wissenschaft und Literatur durchgeführt und danach noch mehrfach in Dresden und Berlin über kulturelle, ökonomische und politische Themen gelesen

29 Zum Erfolg seiner Vorlesungen, die er im Sommersemester 1794 unter dem Titel „Moral für Gelehrte“ zum ersten Mal hielt, schreibt Fichte am 26. Mai 1794 an seine Frau: „Verwichnen Freitag hielt ich meine erste öffentliche Vorlesung. Das gröste Auditorium in Jena war zu enge; der ganze Hausflur, der Hof stand voll, auf Tischen, u. Bänken standen sie einander auf den Köpfen.“ Zit. nach dem Kommentar in Fichte 1997: 931.

30 Schiller 1993b: 749-767. 
hatte. ${ }^{31}$ Alle diese Vorlesungen waren auch publiziert worden, doch entscheidend ist für Müller, dass sie ursprünglich tatsächlich vor Publikum gehalten wurden, dass sie ihre erste Wirkung nicht der Schrift, sondern der gesprochenen Sprache verdankten.

\section{Das Medium der Gegenwart}

Das Elend der deutschen Sprache besteht nach Müller darin, dass die öffentliche Kommunikation in den deutschsprachigen Ländern „stumm“ (297) verläuft; zum einen, weil sie in die tote Schrift gebannt sei, zum andern, da das Deutsche neben dem im höheren gesellschaftlichen Verkehr gepflegten Französisch im eigentlichen Sinne nicht mehr zu Wort komme. So fragt er rhetorisch: „Können wir Deutsche von Beredsamkeit sprechen, nachdem längst aller höhere Verkehr bei uns stumm und schriftlich oder in einer auswärtigen Sprache getrieben wird?“ (297) Unter solchen Bedingungen habe sich keine deutsche Beredsamkeit, sondern - wie Müller mit einem reizvollen Neologismus formuliert - nur eine deutsche „Beschreibsamkeit“ (382) entwickeln können, eine Schreibkultur, die jeden Bezug zur lebendigen Rede verloren habe. Besonders dramatisch scheint Müller dieser Missstand auch in der deutschen Wissenschaft zu sein, wo die Kultur des gesprochenen Worts gänzlich verdrängt worden sei durch einen bloß noch „telegraphischen Verkehr, den die Philosophen und Gelehrten der einzelnen Sekten, jeder in seiner besonderen Terminologie, über die weite Fläche von Deutschland miteinander treiben“ (298). Und schlimmer noch: Die deutschen Gelehrten haben mit dem Reden auch die Kunst der Adressierung eines Gegenübers verlernt, so dass sie in ihren Büchern, wie Müller bitter konstatiert, „eigentlich niemanden anreden, sondern in sich selbst hineinsprechen“ (298). Das Reden über Wissenschaft sei gänzlich verdrängt worden durch ein autistisches Schreiben, durch „ein einförmiges Rauschen der Bücherblätter in einsamen Gemächern“ (328).

Die Schuld aber an dieser Misere ist nach Müller in der Entwicklung des Buchdrucks zu suchen, dessen Auswirkungen in Deutschland, anders als in England und Frankreich, nicht durch gewisse Gegenkräfte einer weiterhin gepflegten Rhetorikkultur kompensiert worden seien. Die „Buchdruckerkunst“ habe eine „Verwirrung und Verkehrung im Reiche der Geister“ angerichtet

31 Vgl. allgemein zum Phänomen der Vorlesungen der genannten Autoren Riedl 1997: 214-294; für eine Übersicht zu Müllers Vorlesungstätigkeit vgl. den Kommentar in Müller 1967c: $674 \mathrm{f}$. 
(327), und zumal durch die ungeheure Zunahme an Publikationen im Laufe des 18. Jahrhunderts sei ein ganz „unnatürliche[r] Zustand“ eingetreten, durch den „die Erziehung der ganzen gegenwärtigen Generation verwirrt worden ist“ (410). Diesen „unnatürlichen Zustand“ kontrastiert Müller mit der Zeit vor der Erfindung des Buchdrucks: „In den Zeiten vor dieser [...] Erfindung wurde die Kunst der Schrift nur angewendet für die Abwesenden und Nachkommen: für die Gegenwärtigen hingegen, für die Zeitgenossen, für alles, was man mit seiner Brust und Stimme erreichen konnte, galt die lebendige Rede“ (327, Hvh. P.S.).

Damit wird mit phonozentristischem Einschlag eine Vergangenheit heraufbeschworen, in der die zeitliche Gegenwart noch mit der räumlichen kongruent war. Es kommt mithin - durchaus zeittypisch - zu einer affirmativen Bezugnahme auf die mittelalterliche Vorlesungskultur. ${ }^{32}$ Und in der Idealisierung dieser Vergangenheit der Kommunikation scheint sich auch ein reaktionärnostalgischer Zug in der Argumentation Müllers bemerkbar zu machen. Doch dass der Weg zurück in die idealisierte Vergangenheit in der Gegenwart des Jahres 1812 keine Option ist, ist Müller bewusst. Vielmehr geht es ihm darum, den unmittelbaren Gegenwartsbezug des Redens über Wissenschaft unter den irreversiblen Bedingungen des Buchdrucks, dessen Vorteile er durchaus kennt, wieder zur Geltung kommen zu lassen; und zwar dadurch, dass nun - wie er in einer normativen Wendung postuliert - nur noch gedruckt werden soll, was zuvor in der lebendigen, gesprochenen Sprache als dem eigentlichen Medium der Gegenwart und der Gegenwärtigkeit vorgetragen worden ist; ${ }^{33}$ ein Postulat, das zugleich auch den drohenden performativen Widerspruch in seiner eigenen Kommunikationspraxis entkräften soll, in der er ja keineswegs darauf verzichtete, seine Vorlesungen und Reden post festum - zunächst auszugsweise in Zeitschriften und dann auch integral in Buchform - zu publizieren. ${ }^{34}$

32 Vgl. zu dieser, auch z. B. bei Fichte auszumachenden, positiven Bezugnahme auf den mittelalterlichen Vorlesungsbetrieb Hambsch 2009: 1199f. In diesen Zusammenhang gehört auch Müllers emphatische Forderung nach einer Aufwertung von Handschrift und Manuskript gegenüber dem Buchdruck, vgl. dazu Müller 1967a: 414-417.

33 Vgl. in diesem Zusammenhang z. B. auch 377: „Also wirf die Feder beiseite, wo sie nicht hingehört; denke nicht früher an die Nachwelt, als bis du die Gegenwart besorgt, schreibe nicht eher, bis du reden kannst, damit du zuletzt wenigstens Gesprochenes niederschreibst [. . .].“ Vgl. allgemein zur Rolle der mündlichen Kommunikation für die Gemeinschaftsbildung bei Müller Matala de Mazza 1999: 298-339.

34 Im Falle der Zwölf Reden ist es so, dass die 2., 3., 6. und 9. Rede bereits in den Jahren 1814/ 15 in der Zeitschrift Friedensblätter publiziert wurden. Vgl. für die Einzelnachweise die Angaben im Kommentar zu Müller 1967c: 606-610. Vollständig erschienen die Zwölf Reden, wie erwähnt, erst 1816 bei Göschen in Leipzig. 
Würde Müller in diesem Zusammenhang freilich nur ein normatives Postulat formulieren, stellte sich immer noch die Frage, ob er damit seine eigenen, in der „Verzauberung der Schrift“ (365) gefangenen Zeitgenossen erreichen könne. Schließlich besteht für jeden ,Unzeitgemäßen“ die Gefahr, dass er unter den Bedingungen seiner Gegenwart nicht rezipiert werden kann - es sei denn, es gäbe eine Entwicklung in jener Gegenwart, die auch andere das Unzeitgemäße als zeitgemäße Avantgarde erkennen lässt. Und tatsächlich glaubt Müller, bereits Ansätze zu einer solchen Entwicklung im Reden und Schreiben über Wissenschaft ausmachen zu können. Denn wenn man genauer hinschaue, sei der Höhepunkt des chaotischen Publikationswesens ohnehin schon überschritten. Es sei zu einer „Antiquität“ (411) geworden, da niemand mehr alles lesen könne, was geschrieben werde, und deshalb eine notwendige Selektion eingesetzt habe; ${ }^{35}$ eine Entwicklung, die Müller in einem prophetischen Futur vorträgt, das die eigene Gegenwart und die jüngste Vergangenheit immer schon mit einbegreift:

[I]n dem Maße, als sich die Anzahl der Schreibenden der Anzahl der Lesenden nähert oder als jene diese übertrifft, legt sich das Schreiben von selbst, [. . .] nur die ganz großen Autoritäten, die in allen Jahrhunderten haben sprechen dürfen, werden durchdringen und allein reden: ihnen werden die Vorteile der Buchdruckerkunst zugute kommen. [...] Durch die Buchdruckerkunst wird in der Folge der Zeiten nur wirken können, der, was er sagen wird, vorher ausgesprochen, erlitten, erlebt haben wird [...]. Es wird nur gedruckt werden, was gesprochen worden: die Druckerkunst wird nur gelten als eine dienende Beihilfe für die eigentliche rednerische Tat. (411f.)

Die eigentliche rednerische Tat ist also, nach Müller, wieder an der Zeit; sie ist es, die auf die eigene Gegenwart zu wirken vermag, und zwar gerade deshalb, weil sie da vollbracht wird, wo ein Redner in Gegenwart eines ganz konkreten Publikums spricht, auf das er immer wieder neu aus dem Moment heraus zu reagieren vermag; kurz, wo er der Forderung nach Geistesgegenwart gerecht wird.

35 Vgl. auch 322: „Ich wünsche Deutschland Glück, daß jenes Schrift- und Formelwesen allmählich zerfällt, daß das Ansehn der Druckerpresse durch den Mißbrauch allmählich abnimmt, daß die Liebhaber dieses Unwesens von den Zeitumständen [den Auswirkungen der napoleonischen Kriege] mehr und mehr mit Auswahl zu kaufen und anstatt zu lesen lieber zu sprechen genötigt werden [...].“ 


\section{Geistesgegenwart}

Wenn von der (räumlichen) Gegenwart des Publikums die Rede ist, muss zugleich, und vielleicht sogar zuerst, auch von der (räumlichen) Gegenwart des Autors als Redner gesprochen werden. Denn gerade im Blick auf diesen Aspekt der Rhetorik als „gegenwärtigste[r]“ Kunst wird eine Bedeutungsdimension des Gegenwartsbegriffs besonders gut sichtbar, die bis ins frühe 19. Jahrhundert noch eine wichtige Rolle spielte, dann aber verblasste. Die Gegenwart einer Person bedeutete bis dahin nicht nur - wie eingangs der Prägnanz halber gesagt deren bloße räumliche Anwesenheit, sondern konnte auch eine besondere Wirkung und Ausstrahlung dieser Person implizieren. ${ }^{36}$ Es ist dies eine Bedeutung, die heute nur noch im Wort ,Präsenz' nachhallt, wenn zum Beispiel von der ,Bühnenpräsenz' eines Schauspielers die Rede ist. Genau dieser semantische Aspekt des Gegenwartsbegriffs aber spielt in Müllers Konzept der Beredsamkeit noch eine zentrale Rolle. Der gute Redner wirkt unmittelbar durch seine schiere Gegenwart. Diese kommt freilich nur dann unverstellt zur Geltung, wenn er frei, aus dem Moment heraus spricht und sich nicht auf ein vorbereitetes Manuskript stützt. Seine Rede kann und soll erst in Gegenwart seines Publikums entstehen, wobei auch bei dessen Gegenwart das Moment der aktiven (Rück-) Wirkung auf den Redner mitzudenken ist. Die wahre Rede lebt also gleichsam von der Wechselwirkung zwischen der Gegenwart des Redners und derjenigen des Publikums, und nur wenn diese Wechselwirkung zustande kommt, entfaltet eine Rede ihre ganze Macht in der (zeitlichen) Gegenwart als dem Moment, in dem sie gehalten wird.

[S]ie [die Rede] ist eigentlich nicht eher da, als bis sie leibhaftig vor denen ausgesprochen wird, die dadurch ergriffen werden sollen. Pronunciatio übersetzt Quintilian das Wort des Demosthenes [für das konkrete Halten einer Rede]; ${ }^{37}$ das deutsche Wort Vortrag bezeichnet nur unvollständig, was er meint. Seine eigentliche Meinung war: es redet ein Gott durch

36 Vgl. dazu Grimm 1897: 2285, wo es heißt, das Wort „gegenwart“ habe nicht nur eine „blosze anwesenheit“ gemeint, „wie es uns [am Ende des 19. Jahrhunderts] begrifflich einschrumpft, sondern zugleich de[n] bereich der lebendigen wirkung [ ]einer person und gewalt [. . . “. Nur vor diesem Hintergrund ist die Definition von ,Gegenwart" bei Adelung verständlich: „Der Zustand, da man durch seine eigene Substanz ohne moralische Mittelursachen, ja ohne alle Werkzeuge an einem Orte wirken kann [...].“ (Adelung 1796: 488)

37 Müller verweist hier auf die - unmittelbar zuvor (365) von ihm selbst zitierte - berühmte Stelle bei Quintilian, wo berichtet wird, dass jemand Demosthenes gefragt habe, was bei einer Rede das Wichtigste sei, und dieser geantwortet habe, es sei die Präsentation der Rede vor dem Publikum (pronuntiatio). Und am zweit- und drittwichtigsten sei ebenfalls der Vortrag. Quintilian 1970: 654 (XI, 3, 6): „[S]i quidem et Demosthenes, quid esset in toto dicendi opere primum interrogatus, pronuntiationi palmam dedit, eidemque secundum ac tertium locum [...].“ 
den Mund des Menschen, wo dieser wirklich redet; und der Gott soll nicht etwa auf die Hörer warten, sondern diese müssen erst zugegen sein, dann erscheint er. - Das ist der Zauber jener unwillkürlichen Beredsamkeit, welche der große Moment selbst herbeiführt; wenige abgerissene Worte, weil sie recht in die Gegenwart hineinfallen, [...] können Wirkungen hervorbringen, welche die absichtliche Redekunst nie erreicht. (366f.)

Die Voraussetzung für das Gelingen dieser „unwillkürlichen Beredsamkeit“ des „improvisierenden Redners“ (333) ist aber, wie Müller formuliert, eine „erhabene Gegenwart des Geistes wie der Organe“ (367). Denn dieser Redner muss eben jederzeit dialogisch auf seine Hörer reagieren, das heißt auf ihre „leisen Bewegungen des Blickes oder der Augenbrauen“, auf ihr „leise[s] Zucken der Muskeln“, ihr „unmerkliche[s] Lächeln“, ihre „Atemzüge[ ]“ und ihre „Pulsschläge[ ]“ (334). - Kurz: die im emphatischen Sinne lebendige Redekunst ist eine Kunst der „allmählichen Verfertigung der Gedanken beim Reden“, bei der das „Zucken einer Oberlippe“ eines Zuhörers oder dessen „zweideutiges Spiel an der Manschette“ unter Umständen „den Umsturz der Ordnung der Dinge“ bewirken kann. ${ }^{38}$ Und tatsächlich ist dieser Anklang an Kleist kein Zufall, denn nur wenige Jahre zuvor hatte Müller (wahrscheinlich) den einschlägigen Aufsatz seines Freundes redigiert. ${ }^{39}$

Auch in diesem Punkt drohte Müller freilich ein performativer Selbstwiderspruch, denn in seinem Reden über die Wissenschaft vom Reden vertraute er selbst keineswegs auf die Inspiration des Augenblicks und auf seine Geistesgegenwart. Vielmehr las er, genau wie Friedrich Schlegel vor ihm, aus einem zuvor sorgfältig konzipierten Manuskript - freilich offenbar gekonnter als jener, wenn man dem Bericht der Schriftstellerin und Salonière Caroline Pichler glauben darf, die in jenem Frühjahr 1812 beide Romantiker gehört hat:

Er [Müller] las mit gemäßigter, nicht ganz von Manier freier Stimme, zusammenhängend, in geregeltem Flusse aus seinem Manuscripte, das vollkommen vor der Lesung geordnet zu seyn schien. Schlegel hingegen, obwohl sein Vortrag lebendig und natürlicher als der Müller's war, mußte oft in seinen Blättern den Zusammenhang nachsuchen, die Einschiebsel nachholen, manchen Satz wiederholen. Das war nun freilich etwas störend, und dies wußte Müller zu vermeiden. ${ }^{40}$

38 Kleist 1985a: 321.

39 Vgl. dazu den Kommentar in Kleist 1985b: 925. Auch wenn die Redaktionsspuren in Kleists erst posthum publiziertem Text nicht von Müller stammen sollten, darf davon ausgegangen werden, dass dieser die Gedanken seines Freundes aus der gemeinsamen Dresdener Zeit kannte. Zur Parallele zwischen Kleist und Müller in dieser Hinsicht vgl. Riedl 1997: 123-154. Riedl arbeitet dann auch die Differenzen heraus, die sich in den Ausarbeitungen dieses gemeinsamen Grundkonzepts bei Kleist und Müller ergeben. Vgl. auch Mionskowski 2013.

40 Pichler 1844: 235f. Einige Zeugnisse von Zeitgenossen wie Dorothea Schlegel, Theodor Körner und Wilhelm von Humboldt, die teilweise beide Vorlesungszyklen gehört haben, sind 
Obschon Müller also mit seinem Manuskript - das übrigens später bei der Publikation, genau wie das Schlegels, mehr oder weniger unverändert abgedruckt wurde ${ }^{41}$ - beim Vorlesen besser zurecht kam als sein Kollege, konnte er als Redner seinem eigenen Ideal nicht gerecht werden. Und das war ihm durchaus bewusst, wie mehrere Passagen in den Zwölf Reden zeigen, in denen er das Publikum bittet, „gütigst für den Augenblick uns lesende, schreibende Redner und alle gelehrte Barbarei und alle Stubenangewöhnungen unserer Zeit“ $\mathrm{zu}$ vergessen (333), oder in denen er selbstkritisch von der „Verlegenheit“ spricht, „die jeder empfindet, der eine vorbereitete Rede vor einer Versammlung aussprechen will“ (365). Diese Verlegenheit rührt daher, dass das vorbereitete Manuskript die im lebendigen Reden über Wissenschaft notwendige Spontaneität verhindert; es hemmt gleichsam die Geistesgegenwart des Redners und verhindert so die Gegenwart eines die Redesituation durchwaltenden höheren Geistes.

\section{Die Gegenwart des Geistes}

Die deutsche Sprache wird, wie bereits angedeutet, spätestens nach der Schlacht bei Jena und Auerstedt im Jahre 1806 zum Politikum. Sie ist das, was den Besiegten geblieben ist, und wird zum emphatisch beschworenen Medium für einen deutschen Sprachnationalismus. Solange diese Sprache aber eben, wie Müller meint, eine stumme Büchersprache bleibt, taugt sie nicht für ein lebendiges Gemeinschaftsgefühl: „Es ist ein schlechter Trost, den wir auf allen Straßen hören müssen, daß uns nämlich statt Deutschland das Instrument der deutschen Sprache verblieben sei. Was ist diese tote Schriftsprache ohne das lebendige Gespräch und ohne die deutsche Rede, die daraus hervorwachsen sollte.“ (322)

Der enge Bezug zwischen deutschem Gespräch und deutscher Rede, der in diesem Zitat anklingt, ist von zentraler Bedeutung für Müllers Konzept

\footnotetext{
zusammengestellt im Kommentar zu Müller 1967c: 458-461. Alle drei Genannten urteilten kritisch über Müllers Vorlesungen, wobei im Urteil Dorothea Schlegels sehr deutlich der Ärger darüber zum Ausdruck kommt, dass Müller mit seiner „crême[!] fouettée“ noch mehr Erfolg hatte beim Publikum als ihr Mann: Im Grunde seien Müllers Vorträge doch nur „Nachlesungen“ zu den Vorlesungen Friedrich Schlegels (ebd.: 459). Für Humboldt waren beide Vorlesungszyklen nur „sophistische Rhetorik“ (ebd.: 460).

41 Müller hat seinen Text im Druck von 1816 lediglich mit zwei Fußnoten ergänzt, die Stellen erklären, die unmittelbar auf Ereignisse des Jahres 1812 Bezug nehmen, vgl. 299, 323. Schlegel sagt in der Einleitung zur Buchfassung seiner Vorlesungen, er habe diese so geschrieben, dass sie auch für den Druck geeignet seien, vgl. Schlegel 1815: XII.
} 
einer deutschen Beredsamkeit. Denn wie er schon in der zweiten und dritten seiner Reden darlegt, ist jede wahre Rede im Grunde ein Gespräch. ${ }^{42}$ Sie lebt, wie gerade dargelegt, von der Wechselwirkung zwischen dem Redner und seinen Hörern, welch letztere durch ihre schiere Gegenwart die Entwicklung jeder Rede mitprägen. Das Gegenüber der Hörer regt den Redner dazu an, nicht nur seinen eigenen Standpunkt, sondern auch mögliche Einwände dagegen zu antizipieren. So tut der Redner, wie Müller ausführt, nichts anderes, als „was in jedem wahren Gespräch bewußtlos geschieht“ (319):

[E]r stellt dar 1. den Streit zweier ganz eigentümlichen und verschiedenartigen Naturen, 2. das Gemeinschaftliche, Höhere, was in dem lebendigen Gespräch unsichtbar wie ein Schutzgeist des Gesprächs oder wie die Grundharmonie in der Musik zwischen den beiden Sprechenden waltet. Eine Rede ist also nichts anders als ein abgeschlossenes Gespräch, welches in allen seinen wesentlichen sichtbaren und unsichtbaren Teilen durch den Mund Eines Menschen an die Welt tritt. Der Redner vereinigt drei Personen in sich, zuvörderst die beiden Sprecher des Gesprächs in ihrer eigentümlichen Farbe und Manier, dann aber beide gedämpft, veredelt sichtbar und unsichtbar versöhnt durch eine dritte höhere Person [...]. (319f.)

Das ist gleichsam die rhetorische Applikation von Müllers einige Jahre zuvor entwickelter Lehre vom Gegensatz (1804). Was allerdings mit jener Figur des Dritten genau gemeint ist, ist zunächst nicht ganz klar erkennbar. Im Anschluss an die eben zitierte Passage nennt Müller sie die „Seele des Redners, die über dem Streite der Glieder thront“ (319); an anderer Stelle, bei seinen anglophilen Ausführungen zur britischen Parlamentsrhetorik, ist jenes Dritte der ,Geist der englischen Verfassung‘, die - was in diesem Zusammenhang wichtig ist - keine im Schriftmedium niedergelegte Konstitution ist. ${ }^{43}$ Die wichtigste Bedeutung jenes Dritten für die deutsche Beredsamkeit wird aber in den abschließenden Reden deutlich, wo es als Geist der deutschen Nation und Geist des Christentums erkennbar wird. Die Beredsamkeit, die im Zeichen dieses Geistes steht, ist nach Müller nicht mehr eine, die - wie die heidnisch-antike Rhetorik - auf die gewaltsame Überwindung des Gegners angelegt ist, sondern eine, die ihr Gegenüber ernst nimmt und sich selbst demütig jener höheren Macht unterwirft (431); ganz im Sinne der paulinischen Beredsamkeit, die sich nicht „überredende[r] Worte menschlicher Weisheit“

42 Vgl. 310-336. Vgl. zu diesem Gedanken z. B. auch Schleiermacher 1996: 372: „[D]er Kathedervortrag der Universität muß allerdings, weil er Ideen zuerst zum Bewußtsein bringen soll, [. . .] die Natur des alten Dialogs haben, wenn auch nicht seine äußere Form [...].“

43 Vgl. dazu 314 sowie Müllers Ausführungen zu Edmund Burke, der in seinen Reden jenen Geist zur Erscheinung kommen lasse und insofern zum „Stellvertreter des unsichtbaren Englands“ geworden sei (375). 
bedienen will. ${ }^{44}$ Und sie ist eine Rhetorik der Pastoralmacht, deren Adressierungsstruktur sich dadurch auszeichnet, dass durch sie alle gemeinsam und zugleich jeder als Einzelner angesprochen wird - omnes et singulatim, um es mit dem Titel von Michel Foucaults einschlägigen Tanner Lectures zu formulieren. ${ }^{45}$ In den Worten Adam Müllers lautet diese pastorale Formel: „, [I]n jedem einzelnen Menschen werden unendliche viele, ja alle angeredet“ (443).

Die vom Geist des Christentums geprägte Rhetorik Müllers ist getragen von einem Gefühl von „Freiheit in dem Gehorsam, des Stolzes in der Unterwerfung“ (432). Und gerade von dieser christlichen Denkfigur her ergibt sich nun für Müller eine glänzende Aussicht auf die Zukunft der deutschen Beredsamkeit und der von Napoleon gedemütigten deutschen Nation:

Hat sich in allen Klagen über das Verstummen meines Jahrhunderts, zumal meines deutschen Vaterlandes, ein gewisses Gefühl der Freiheit, ich möchte sagen ein Vorgefühl künftiger Beredsamkeit, besonders aber ein Gefühl der Beruhigung in dem Schmerz, des Sieges in der Niederlage merken lassen? darauf kommt es an. Von dieser Seite, und es ist die einzige wesentliche, steht das Land, welches mich erzogen hat, über allen andern.

Das Gefühl der freien Dienstbarkeit und des würdevollen Gehorsams ist das Palladium der Welt: Deutschland ist dessen Pflege anvertraut. (433) 46

Doch wo ist für Müller in seiner Gegenwart der Ort, an dem im gedemütigten Deutschland jenes Palladium durch die geschilderte, im eigentlichen Sinne zukunftsträchtige Beredsamkeit gepflegt werden soll? Es ist nicht ein noch zu gründendes Parlament, wie man nach den enthusiastischen (und für Müller nicht ungefährlichen) Ausführungen zur politischen Rhetorik in England glauben könnte. ${ }^{47}$ Vielmehr ist es - und damit schließt sich der Bogen zur

44 1. Kor 2,4: „[U]nd meine Predigt geschah nicht mit überredenden Worten menschlicher Weisheit [ouk en peithois sophias logois], sondern in Erweisung des Geistes und der Kraft [...]. Vgl. zur antipersuasiven christlichen Rhetorik Hagemann 2014: 715-723, zur PaulusStelle 715 .

45 Foucault 1981: 223-254. Vgl. zum Begriff der „Pastoralmacht“ als einer Macht, die auf pastoraler Seelenführung beruht, wie sie vor allem in der jüdisch-christlichen Tradition entwickelt wurde, Foucault 2006: 184-194, 201-330. Vgl. allgemein zur gouvernementalen Dimension der Rhetorik in der politischen Romantik Schnyder 2014: 663-679.

46 Vgl. auch 449: „Um dieses [christlichen] Geistes willen kann man festiglich glauben, daß die Sprache der Besiegten länger leben werde als die der Sieger, und in diesem Sinne dann dreist verkünden, daß, weil die Sprache fortdauern werde, auch das Volk nicht untergehen könne."

47 Zunächst meint Müller, „das britische Parlament“ sei „der einzige Schauplatz echter Beredsamkeit, der diesem unserm stummen Jahrhundert verblieben ist“ (374). Später fügt er aber vorsichtig an: „Ich spreche von England als einem Muster in Sachen der Beredsamkeit [...]: 
Vorlesungsthematik - zwar nicht alleine, aber doch vor allem der Ort, wo über Wissenschaft und insbesondere über Sprache und Poesie geredet wird: Die Schule und die Universität. Denn

[u]nter allen Anwendungen der Beredsamkeit, die in unserer Zeit vorkommen, ist keine wichtiger als die, welche die nächstfolgende Generation, ihre Bildung, Erziehung und Belehrung im Auge hat. [. . . ] Der Erzieher der Jugend [. . .] hat grade jene Jahre des menschlichen Lebens in seiner Disposition, wo die Seele noch mit einer gewissen Frömmigkeit, mit Glauben und Ergebung hinnimmt, was ihr geboten wird, wo sie ein Bedürfnis hat zu empfangen. Das Studium der Sprachen, wie der Poesie und Beredsamkeit, füllt diese Jahre sehr natürlich aus [...]. (434f.)

So wird das wissenschaftliche Reden über Sprache und Literatur bei Adam Müller zu jenem sprachlichen Geschehen, in dem die Gegenwart des christlich-nationalen Geistes am intensivsten erfahren werden kann. Und der Deutschunterricht an der Schule und die Vorlesung über deutsche Sprache und Literatur an der Universität werden damit $\mathrm{zu}$ einem herausgehobenen Ort pastoral-gouvernementaler Intervention.

\section{Literatur}

Adelung, Johann Christoph (1796): Gegenwart. In: Ders., Grammatisch-kritisches Wörterbuch der Hochdeutschen Mundart. Zweyte vermehrte und verbesserte Auflage, Zweyter Theil. Leipzig: Breitkopf, 488.

Campe, Johann Heinrich (1808): Gegenwart. In: Wörterbuch der Deutschen Sprache. Zweiter Theil. Braunschweig: Schulbuchhandlung, 265.

Dick, Alexander \& Angela Esterhammer (Hrsg.) (2009): Spheres of Action. Speech and

Performance in Romantic Culture. Toronto: University of Toronto Press.

Fichte, Johann Gottlieb (1997): Reden an die deutsche Nation. In: Schriften zur angewandten

Philosophie. Bd. 2. Hrsg. von Peter Lothar Oesterreich. Frankfurt a.M.: Deutscher Klassiker Verlag, 539-788.

Foucault, Michel (1981): Omnes et singulatim. Towards a Critique of ,Political Reason‘. In: Sterling M. McMurrin (Hrsg.), The Tanner Lectures on Human Values. Bd. 2. Salt Lake City, Cambridge: University of Utah Press, 223-254.

aber wehe dem Staate, der die Äußerlichkeiten britischer Verfassung sich anzueignen suchte: er ist schlimmer daran als die, welche hartnäckig bei dem beharren, was sie aus sich selbst sind, wie wenig es auch sei.“ (428) Trotz dieser Vorsicht nahm der Polizeispitzel in Müllers Vorlesung Anstoß an Müllers Anglophilie (und der damit einhergehenden Frankreichkritik). Der Kaiser persönlich verfügte aufgrund der entsprechenden Berichte, dass Müller, „falls er neue Vorlesungen zu halten willens wäre, vor jeder Vorlesung die Handschrift der Polizei und Zensurhofstelle zur Durchsicht vorlegen soll“, zit. nach dem Kommentar in Müller 1967c: 462. Dazu kam es nicht, da Müller keine weiteren Vorlesungen mehr hielt. 
Foucault, Michel (2006): Sicherheit, Territorium, Bevölkerung. Geschichte der

Gouvernementalität I. Hrsg. von Michel Sennelart. Übers. von Claudia Brede-Konersmann und Jürgen Schröder. Frankfurt a.M.: Suhrkamp.

Grimm, Jacob \& Wilhelm Grimm (1897): gegenwart. In: Dies. (Hrsg.), Deutsches Wörterbuch. Bd. 5. Leipzig: S. Hirzel, 2281-2300.

Hambsch, Björn (2009): Vorlesung. In: Gert Ueding (Hrsg.), Historisches Wörterbuch der Rhetorik. Bd. 9. Berlin: De Gruyter 2009, 1194-1209.

Hagemann, Tim (2014): Antipersuasive und Aristotelische Rhetorik. In: Gregor Kalivoda \& Gert Ueding (Hrsg.), Wege moderner Rhetorikforschung. Klassische Fundamente und interdisziplinäre Entwicklung. Berlin, Boston: De Gruyter, 715-723.

Herrmann, Britta (2015): Für eine wahrhaft deutsche Kunst und Rede. Friedrich Schlegel und Adam Müller. In: Athenäum 25, 227-248.

Kant, Immanuel (1974): Kritik der Urteilskraft. In: Werkausgabe. Bd. 10. Hrsg. von Wilhelm Weischedel. Frankfurt a.M.: Suhrkamp.

Kleist, Heinrich von (1985a): Sämtliche Werke und Briefe. Hrsg. von Helmut Sembdner. Bd. 2. 8. Aufl. München: Hanser.

Kleist, Heinrich von (1985b): Über die allmähliche Verfertigung der Gedanken beim Reden. In: Sämtliche Werke und Briefe. Hrsg. von Helmut Sembdner. Bd. 2. 8. Aufl. München: Hanser, 319-324.

Koehler, Benedikt (1980): Ästhetik der Politik. Adam Müller und die politische Romantik. Stuttgart: Klett-Cotta.

Koselleck, Reinhart (1989): Vergangene Zukunft. Zur Semantik geschichtlicher Zeiten. Frankfurt a.M.: Suhrkamp.

Lehmann, Johannes F. (2016): „Ändert sich nicht alles um uns herum? Ändern wir uns nicht selbst?“ Zum Verhältnis von Leben, Zeit und Gegenwart um 1770. In: Benjamin Brückner, Judith Preiß \& Peter Schnyder (Hrsg.), Lebenswissen. Poetologien des Lebendigen im langen 19. Jahrhundert. Freiburg i.Br.: Rombach, 51-73.

Luhmann, Niklas (1980): Temporalisierung von Komplexität. Zur Semantik neuzeitlicher Zeitbegriffe. In: Ders., Gesellschaftsstruktur und Semantik. Studien zur Wissenssoziologie der modernen Gesellschaft. Bd. 1. Frankfurt a.M.: Suhrkamp, 235-300.

Matala de Mazza, Ethel (1999): Der verfaßte Körper. Zum Projekt einer organischen Gemeinschaft in der Politischen Romantik. Freiburg i.Br.: Rombach.

Mercier, Louis-Sébastien (1773): Du théâtre, ou nouvel essai sur l'art dramatique. Amsterdam: Van Harrevelt.

Mercier, Louis-Sébastien (1776): Neuer Versuch über die Schauspielkunst. Aus dem Französischen [von Heinrich Leopold Wagner]. Mit einem Anhang aus Goethes Brieftasche. Leipzig: Schwickert.

Mionskowski, Alexander (2013): „Jedwede Kunst der Rede ward erschöpft“. Heinrich von Kleist, Adam Müller und die Aporien der Beredsamkeit im Trauerspiel Penthesilea. In: Hans Richard Brittnacher \& Irmela von der Lühe (Hrsg.), Risiko - Experiment Selbstentwurf. Kleists radikale Politik. Göttingen: Wallstein, 56-82.

Müller, Adam (1967a): Zwölf Reden über die Beredsamkeit und deren Verfall in Deutschland. Gehalten zu Wien im Frühlinge 1812. In: Kritische, ästhetische und philosophische Schriften. Bd. 1. Hrsg. von Walter Schroeder \& Werner Siebert. Neuwied, Berlin: Luchterhand, 293-451. 
Müller, Adam (1967b): Vorlesungen über die deutsche Wissenschaft und Literatur. In: Kritische, ästhetische und philosophische Schriften. Bd. 1. Hrsg. von Walter Schroeder \& Werner Siebert, Neuwied, Berlin: Luchterhand, 11-137.

Müller, Adam (1967c): Kritische, ästhetische und philosophische Schriften. Bd. 2. Hrsg. von Walter Schroeder \& Werner Siebert, Neuwied, Berlin: Luchterhand.

o.A. (1735): Gegenwart. In: Großes vollständiges Universal-Lexicon aller Wissenschaften und Künste. Hrsg. von Johann Heinrich Zedler. Bd. 10. Halle: Zedler, 594.

o.A. (1812): Wissenschaftliche und Kunstnachrichten. In: Oesterreichischer Beobachter Nr. 135, 14. Mai 1812, $587 \mathrm{f}$.

o.A. (1939): Gegenwart. In: Alfred Götze \& Walther Mitzka (Hrsg.), Trübners Deutsches Wörterbuch. Bd. 3. Berlin: De Gruyter, 58 f.

o.A. (2005): gegenwart, gegenwurt [Subst.]. In: Ulrich Goebel, Oskar Reichmann \& Anja Lobenstein-Reichmann (Hrsg.), Frühneuhochdeutsches Wörterbuch. Bd. 6. Lieferung 2. Berlin: De Gruyter, $516 \mathrm{f}$.

Oesterle, Ingrid (1985): Der Führungswechsel der Zeithorizonte in der deutschen Literatur. Korrespondenzen aus Paris, der Hauptstadt der Menschheitsgeschichte, und die Ausbildung der geschichtlichen Zeit ,Gegenwart‘. In: Dirk Grathoff (Hrsg.), Studien zur Ästhetik und Literaturgeschichte der Kunstperiode. Frankfurt a.M.: Peter Lang, 11-76.

Quintilian (1970): Institutionis oratoriae libri duodecim. Tomus II: Libri VII-XII. Hrsg. von Michael Winterbottom. Oxford: Oxford University Press.

Pichler, Caroline (1844): Denkwürdigkeiten aus meinem Leben. Bd. 2: 1798-1813. Wien: Pichler.

Polaschegg, Andrea (2017): Geschichte der alten und neuen Literatur. In: Johannes Endres (Hrsg.), Friedrich Schlegel Handbuch. Leben - Werk - Wirkung. Stuttgart: J.B. Metzler, 224-232.

Riedl, Peter Philipp (1997): Öffentliche Rede in der Zeitenwende. Deutsche Literatur und Geschichte um 1800. Tübingen: Niemeyer.

Schelling, Friedrich Wilhelm Joseph (1803): Vorlesungen über die Methode des academischen Studiums [gehalten 1802 in Jena]. Tübingen: Cotta.

Schiller, Friedrich (1993a): Wallenstein. In: Sämtliche Werke. Bd. 2: Dramen 2. Hrsg. von Gerhard Fricke \& Herbert G. Göpfert. 9. Aufl. München: Hanser, 269-547.

Schiller, Friedrich (1993b): Was heißt und zu welchem Ende studiert man Universalgeschichte? Eine akademische Antrittsrede. In: Sämtliche Werke. Bd. 4: Historische Schriften. Hrsg. von Gerhard Fricke \& Herbert G. Göpfert. 9. Aufl. München: Hanser, 749-767.

Schiller, Friedrich (1993c): Was kann eine gute stehende Schaubühne eigentlich wirken? In: Sämtliche Werke. Bd. 5: Erzählungen, Theoretische Schriften. Hrsg. von Gerhard Fricke \& Herbert G. Göpfert. 9. Aufl. München: Hanser, 818-831.

Schlegel, Friedrich (1815): Friedrich Schlegels Geschichte der alten und neuen Litteratur. Vorlesungen gehalten zu Wien im Jahre 1812. Erster Theil. Wien: Schaumburg.

Schleiermacher, Friedrich (1996): Gelegentliche Gedanken über Universitäten in deutschem Sinn. Nebst einem Anhang über eine neu zu errichtende. In: Schriften. Hrsg. von Andreas Arndt. Frankfurt a.M.: Deutscher Klassiker Verlag, 335-438.

Schnyder, Peter (2005): Rhetorik [18./19. Jhdt.]. In: Gert Ueding (Hrsg.), Historisches Wörterbuch der Rhetorik. Bd. 7. Tübingen: Niemeyer, 1468-1472 und 1523-1529.

Schnyder, Peter (2014): Rhetorik und Gouvernementalität in der politischen Romantik. In: Gregor Kalivoda \& Gert Ueding (Hrsg.), Wege moderner Rhetorikforschung. Klassische Fundamente und interdisziplinäre Entwicklung. Berlin, Boston: De Gruyter, 663-679. 